\title{
The prediction research of tool VB value based on Principal Component Analysis and SVR
}

\author{
NIE Peng, HE Chao, XU Liang, CUI Kai-qi \\ School of Mechanical and Electronic Engineering, Shenyang Aerospace University, Shenyang, \\ Liaoning 110136, china \\ email: niehit@163.com, bemail: luomin120223@163.com
}

\begin{abstract}
Keywords: support vector regression(SVR);genetic algorithm; principal component analysis ( $P$ CA);forecast VB value
\end{abstract}

\begin{abstract}
According to the amount of tool wear prediction problems, online prediction of tool wear model is established based on the theory of support vector regression (SVR) regression. The acoustic emission signals and current signals are, respectively EEMD decomposed and wavelet packet decomposed to get the energy values, which are combined with the spindle speed, feeding, and back engagement to form the original feature vectors. By principal component analysis for data processing, the principal elements as the Support Vector Regression (SVR) optimized by genetic algorithms are inputted. The results show that this model has high precision, fast operation.
\end{abstract}

In the tool processing, the acoustic signals and current signals gained by sensor contain abundant processing information, which can reflect the variations of tool wear. As a very promising prediction technology, support vector regression is a model identification method based on statistical learning theory, which shows many advantages in solving the following problems, like small samples, non-linear and high dimensional recognition. The paper adopted support vector machine regression algorithm optimized by genetic algorithm to form a model and predict the VB values of tools ${ }^{[1-2]}$.

\section{The extraction method of signals}

\subsection{EEMD decomposition}

EEMD is a improved method for modal aliasing problems. This method solves the big gest problem of EMD-modal aliasing problem, which extracts the Intrinsic Mode Function (IMF) of aliasing problem. IMF stand for the essence of signal resonant modes, which are used as the basic function determined by the signals and better than the pre-determined ker nel. This is more better for managing non-linear and non-stationary AE signals ${ }^{[3]}$.

The steps of extracting the EEMD decomposition features are as follows.

(1) The original signals are EEMD decomposed to get the IMF components. Then order the sensitive factors by descending, taking the first $\mathrm{n}$ IMF for subsequent processing.

(2) After the ordering, the IMF's first $n$ energy and total energy e:

$$
e_{i}=\sum_{i=1}^{n}\left|y_{i}(t)\right|^{2} \quad(\mathrm{i}=1,2, \ldots, \mathrm{n})
$$

$\mathrm{N}$ represents the number of IMF.

$$
e_{t o l}=\sum_{i=1}^{n} e_{i}
$$

the total energy.

(3) The energy of the first n-sensitive IMF were normalized:

$$
\bar{e}_{i}=\frac{e_{i}}{e_{t o l}} \quad(\mathrm{i}=1,2, \ldots, \mathrm{n})
$$

That feature vector obtained:

$$
x=\left[\bar{e}_{1}, \bar{e}_{2}, \ldots, \bar{e}_{n}\right]
$$


Take the largest eight IMF sensitive factors, and get the feature vector:

$$
x=\left[\bar{e}_{1}, \bar{e}_{2}, \bar{e}_{3}, \bar{e}_{4}, \bar{e}_{5}, \bar{e}_{6}, \bar{e}_{7}, \bar{e}_{8}\right]
$$

Under the condition of the speed $220 \mathrm{r} / \mathrm{min}$, the feed rate $0.2 \mathrm{~mm} / \mathrm{r}$, and cutting depth $0.7 \mathrm{~mm}$ cutting parameters in general CA6140 lathe spindle, the AE signals are EEMD decomposed to get energy, which is shown in Figure 1.

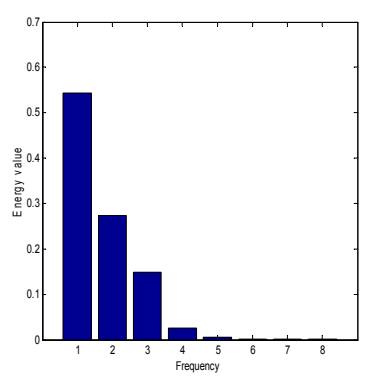

(a) $\mathrm{VB}=0.074 \mathrm{~mm}$

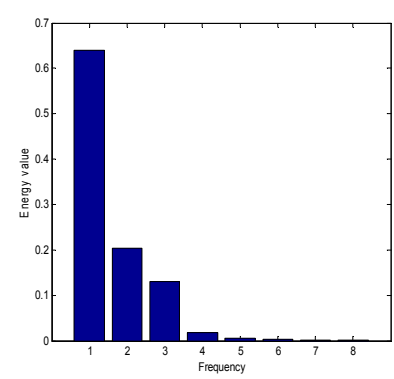

(b) $\mathrm{VB}=0.132 \mathrm{~mm}$

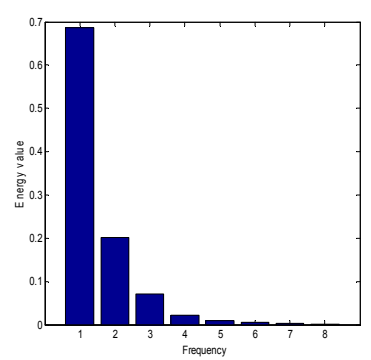

(c) $\mathrm{VB}=0.262 \mathrm{~mm}$

Fig 1 EEMD results of AE signals in the different $\mathrm{VB}$ values

\subsection{Wavelet packet decomposition}

Wavelet packet has the feature of arbitrary multi-scale and avoids the fixed frequency defects during wavelet decomposition, which provides a great choice of frequency analysis, and better reflects the nature and characteristics of the signal[4].

The most important part of wavelet packet decomposition is the selection and identification of wavelet decomposition level. Db8 wavelet packet is widely used. The time-domain waveforms are relatively smooth, and the frequency features are better. The more levels of decompositions are, the more sensitive to changes in signals. But more decomposition levels also mean more computation. Therefore, the paper adopts db8 to wavelet packer decompose the current signals five times.

Under the condition of the speed $355 \mathrm{r} / \mathrm{min}$, the feed rate $0.14 \mathrm{~mm} / \mathrm{r}$, and cutting depth $0.7 \mathrm{~mm}$ cutting parameters in general CA6140 lathe spindle, the AE signals are EEMD decomposed to get energy, which is shown in Fig.2.

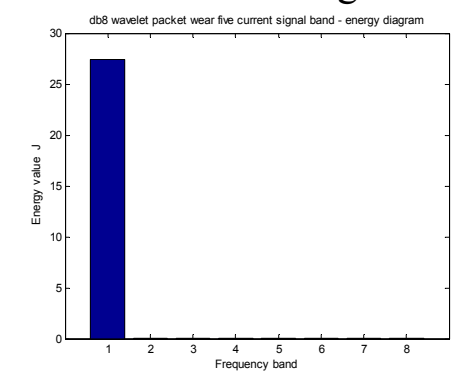

(a) $\mathrm{VB}=0.073 \mathrm{~mm}$

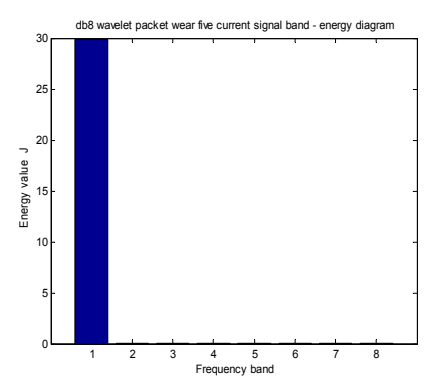

(b) $\mathrm{VB}=0.194 \mathrm{~mm}$

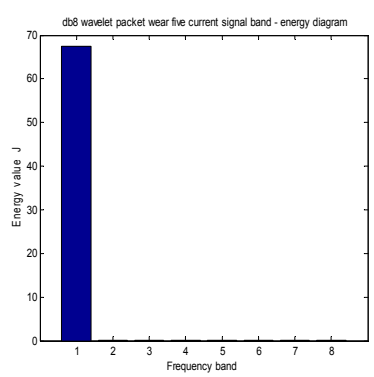

(c) $\mathrm{VB}=0.262 \mathrm{~m}$

Fig 2 wavelet packet decomposed energy diagram of current signals in the different VB values

\section{Principal component analysis}

Principal component analysis was introduced and developed by Pearson and Hostelling. Principal component analysis is a multivariate statistical method to reduce the number of variables into a small number of several main components by dimension reduction. These main components can reflect most of the information of the variables. By linear combining of the original statistics, the information contained by the main components are not overlapped. And there is no correlation between each main component.[5]

\section{Support Vector Machine}

\subsection{The regression modal of support vector machine}

Support vector machine theory is a small sample learning method,SVM has the features of versatility, robustness, and simple[6]. VaPnik introduces the results gained by SVM classification theory through introducing $\varepsilon$-insensitive loss function, and apples them in function fitting. The 
definition of $\varepsilon$-insensitive loss function is as follows.

$$
\theta(y, f(x, a))=\theta\left(|y-f(x, a)|_{\varepsilon}\right)
$$

For a given sample set (yi, $\mathrm{xi}), \mathrm{xi} \in \mathrm{R}^{n}$, $\mathrm{yi} \in \mathrm{R}$, the function is considered.

$$
f(x)=\langle w, \varphi(x)>+b
$$

Where $<\mathrm{w}, \varphi(\mathrm{x})>$ represents $\mathrm{w}$ and $\varphi(\mathrm{x})$ of the inner product, $\mathrm{w} \in \mathrm{R}{ }^{n}, \mathrm{~b} \in \mathrm{R}$. Introducing slack variables $\xi$ i $\left., \quad \xi^{*}{ }_{i}^{*} \geqslant 0, \quad i=1,2, \ldots, n\right)$, structure optimization problem is as follows:

$$
\left\{\begin{array}{l}
\min \frac{1}{2}\|w\|^{2}+C \sum_{i=1}^{n}\left(\xi_{i}+\xi_{i}^{*}\right) \\
\text { s.t. }\left\{\begin{array}{l}
y_{i}-w x_{i}-b \leq \varepsilon+\xi_{i} \\
w x_{i}+b-y_{i} \leq \varepsilon+\xi_{i}^{*}
\end{array}\right.
\end{array}\right.
$$

$\mathrm{C}$ represents the penalty factor, which means the punishment beyond the extent of the error $\varepsilon$ samples. The above formula is the original optimization problem. The objective function of this problem is formed by two parts. The first part is to help the good return hyper plane have good generalization. The second part is to minimize the errors of hyper plane small training set fitting, with the $\varepsilon$-insensitive loss function to judge.

SVR is obtained by solving its dual problem to obtain the optimal solution, which is not directly to solve the original problem.

Since the plots in the kernel function feature space can avoid complex high-dimensional calculations, the choice of the kernel function $\mathrm{K}$ should ensure positive definiteness of the symmetric half.

\section{Experimental process and analysis}

\subsection{Experimental program and procedures}

In the CA6140 lathe, turning YBC carbide blade work piece material is a nickel-base super alloy GH536 with certain blunt standard VB $=0.3 \mathrm{~mm}$. ShengHua RS150 acoustic emission sensors is used and Beijing Forest River industrial production model HZIB-C11-100P2O5 current sensors is used to monitor the state of the cutting tool. Acquisition equipment is the U.S. production data acquisition card NI-779408 with the sampling frequency of 1M Hz. Different 9 kinds of cutting parameters by orthogonal experiment method are grouped. Each group of experiments stops for a while. With the microscope, measure flank wear, draw tool wear curves, and collect acoustic emission signals and current signals related to the tool wear state. Total 90 groups of signal data are collected.

\subsection{The extraction of the features of signals}

Acoustic emission signals 8-band energy decomposed by EEMD and current signal 8 band energy decomposed by db8 wavelet packet are used as feature vectors. Then, together with the cutting speed, feed rate and depth of cutting, 19-dimensional vectors are formed. as a reflection of tool wear feature vector $\mathrm{T}$.

$$
T=[c 1, c 2, \ldots, c 19]
$$

The feature vector $\mathrm{T}$ is first treated standardly, and get M through PCA.

$$
M=(t 1, t 2, t 3)
$$

The original data are reduced from 19 dimensions to 3 dimensions, which largely reduces the managing amount. The 3 main components are used as reflection of tool wear state feature vector and input into support vector machine regression modal.

\subsection{The application of support vector machine}

The kernel function selects RBF kernel function. Use genetic algorithm to optimize the parameters of SVR prediction model[7]. Nuclear parameter range of 0.01 0.1, the step length is 0.01 , punishment factor range of 1 32, step length of 1

(1)SVR forecasting model penalty factor C, G kernel parameter binary coded and randomly 
generated initial population.

(2) Decode each chromosome the of the population to get the value of $\mathrm{C}$ and $\mathrm{G}$. Using part of the data to establish SVR forecasting model to calculate the difference between the predicted and measured values of all data variables with mean relative change in value to get each gene cluster fitness.

(3) Judge whether the stopping criteria of genetic algorithm are met. If they are met, the calculation stops and the optimal parameters are output. Otherwise the execution selection, crossover and mutation are operated to generate new populations, and begin a new generation of heredity.

The final model for the optimal parameters: the penalty factor $\mathrm{C}=2$, the kernel parameter $\mathrm{G}=$ 0.02 .

The previous 72 sets of data modeling are used. The later 18 sets of data are used to be predicted. For comparison, standard BP neural network is used to train and predict these samples and. Prediction results are shown in Tab 1:

Tab 1 Predict results of the part of the test data

\begin{tabular}{|l|l|l|l|l|}
\hline The actual value & SVR Predictive value & $\begin{array}{c}\text { Relative error } \\
\%\end{array}$ & BP Predictive value & Relative error \% \\
\hline 0.107 & 0.1052 & -1.68 & 0.0996 & -6.92 \\
\hline 0.114 & 0.1173 & 2.89 & 0.1032 & -9.47 \\
\hline 0.122 & 0.1181 & -3.19 & 0.1099 & -9.91 \\
\hline 0.143 & 0.1478 & 3.36 & 0.1529 & 6.92 \\
\hline 0.164 & 0.1585 & -3.35 & 0.1755 & 7.01 \\
\hline 0.176 & 0.1829 & 3.97 & 0.1913 & 8.69 \\
\hline 0.194 & 0.2001 & 3.14 & 0.2126 & 9.59 \\
\hline 0.262 & 0.2721 & 3.85 & 0.2416 & -7.78 \\
\hline 0.283 & 0.2925 & 3.36 & 0.2629 & -7.11 \\
\hline
\end{tabular}

From the above figure, the accuracy of support vector machine regression modelis higher than BP neural network, with less than $4 \%$ relative error.

\section{Conclusions}

This paper adopts support vector machine regression theory to predict VB values, and uses the parameters from support vector machine optimized by genetic algorithm. The prediction accuracy increases. After feature vectors are analyzed by components analysis, the dimension of input data is reduced, and the calculation speed increases. It shows that the accuracy of support vector machine is higher with less than $4 \%$ relative error. It can better predict the VB values of tool wear, on the basis of which the tool wear state can be judged.

\section{References}

[1] N.Ghosh, Y.B.Ravi, A.Patra et al. Estimation of tool wear during CNC milling using $\mathrm{n}$ eural network-based sensor fusion[J]. Mechanical Systems and Signal Processing 21 (2007) 466-479.

[2] LI X L. A brief review: acoustic emission method for tool wear monitoring during tur ning[J]. International Journal of Machine Tools \& Manufacture, 2002, 42: 157 -165.

[3] Nie Peng, Xu Hongyao, Liu Xinyu, Li Zhengjiang. EEMD method of tool wear identif ication applications [J]. Sensors and Microsystems, 2012,31 (5): 147-152.

[4] MAO K Z, TAN K C, SER W. Probabilistic neural2net2 work structure determinatio $\mathrm{n}$ for pattern classification [J]. IEEE Tran. NeuralNetworks, 2000, 11 (4) : 101021017.

[5] Tamura M, Tsujita S. A study on the number of principal components and sensitivity of fault detection using PCA[J]. Computers and Chemical Engineering, 2007, 31(9): 1035-1 046.

[6] Wu Jin-hua, Zhou Dawang, Cheng Chunhua. SVM nonlinear identification and control o verview [J]. Tactical Missile Control Technology, 2009,31(2): 28-32

[7] Chen Guo. SVM prediction model time series based on genetic algorithm optimization [J].Journal of Scientific Instrument, 2006,27(9):1080-1084 